\title{
The Inhibitory Role of B7-H4 in Antitumor Immunity: Association with Cancer Progression and Survival
}

\author{
Changjun He, ${ }^{1}$ Haiquan Qiao, ${ }^{1}$ Hongchi Jiang, ${ }^{1}$ and Xueying Sun ${ }^{1,2}$ \\ ${ }^{1}$ The Hepatosplenic Surgery Center, Department of General Surgery, The First Affiliated Hospital of Harbin Medical University, \\ Harbin 150001, China \\ ${ }^{2}$ Department of Molecular Medicine and Pathology, Faculty of Medical and Health Sciences, The University of Auckland, \\ Auckland 1005, New Zealand \\ Correspondence should be addressed to Xueying Sun, k.sun@auckland.ac.nz
}

Received 22 June 2011; Accepted 8 August 2011

Academic Editor: Hiroshi Nakajima

Copyright (๑) 2011 Changjun He et al. This is an open access article distributed under the Creative Commons Attribution License, which permits unrestricted use, distribution, and reproduction in any medium, provided the original work is properly cited.

B7-H4 is one of the most recently identified members of B7 superfamily of costimulatory molecules serving as an inhibitory modulator of T-cell response. B7-H4 is broadly expressed in human peripheral tissues and inducibly expressed in immune cells. The expression of B7-H4 has been observed in various types of human cancer tissues, and its soluble form has been detected in blood samples from cancer patients. However, its precise physiological role is still elusive, as its receptor has not been identified and the expression levels are not consistent. This paper summarizes the pertinent data on the inhibitory role of B7-H4 in antitumor immunity and its association with cancer progression and survival in human patients. The paper also discusses the clinical significance of investigating B7-H4 as potential markers for cancer diagnosis and prognosis, and as therapeutic targets.

\section{Introduction}

Activation of $\mathrm{T}$ lymphocytes requires two independent but mandatory signals. The first signal requires recognition of the major histocompatibility complex (MHC)/antigen on antigen presenting cells (APCs) and corresponding antigenspecific T-cell receptor (TCR) on T cells. On the other hand, the second signal is delivered by the binding of costimulatory molecules and their receptors/ligands. In the absence of the costimulatory signal, the ligation of TCR with the MHC/antigen complex results in disfunction or anergy of $\mathrm{T}$ cells. The typical costimulatory signals are rendered by the molecules of the "classic" B7 family including CD80, CD86, and their receptor $\mathrm{CD} 28$ and cytotoxic T lymphocyte antigen (CTL-4), which could provide positive and/or negative costimulatory signals in initiating T-cell response. Recently, several B7 homologues have been identified, including B7$\mathrm{H} 1, \mathrm{~B} 7 \mathrm{DC}, \mathrm{B} 7-\mathrm{H} 2, \mathrm{~B} 7-\mathrm{H} 3, \mathrm{~B} 7-\mathrm{H} 4$, and $\mathrm{B} 7-\mathrm{H} 6$. B7-H4 (also known as $\mathrm{B} 7 \mathrm{x}$ or $\mathrm{B} 7 \mathrm{~S} 1$ ) is among the most recently identified members of the B7 superfamily. It is broadly expressed in many human tissues and cells, and is shown to regulate adaptive immune response by inhibiting the proliferation, activation, and cytokine production of $\mathrm{T}$ cells, and host innate immune response by suppressing growth of neutrophil progenitors. It is also expressed in many types of human cancers, and has been used as a negative prognostic indicator for many human tumors. Therefore, B7-H4 represents a novel frontier of investigation for understanding the molecular regulation of the immune system and targeting B7-H4 may help to overcome the inhibitory immune network in tumor environments. This paper discusses the inhibitory role of $\mathrm{B} 7-\mathrm{H} 4$ in antitumor immunity, and its association with cancer progression and survival in human patients. It also discusses the clinical significance of investigating $\mathrm{B} 7-\mathrm{H} 4$ as potential markers for cancer diagnosis, and prognosis, and as therapeutic targets.

\section{Structure and Expression Pattern of B7-H4}

B7-H4 was identified by DNA sequence homology with other molecules of the B7 family in 2003 by three laboratories, which designated three different names to the same molecule, that is, $\mathrm{B} 7 \mathrm{~S} 1, \mathrm{~B} 7-\mathrm{H} 4$, and $\mathrm{B} 7 \mathrm{x}$, respectively [13], but now B7-H4 has been most widely used. B7-H4 is a 
type I transmembrane protein and has $20-30 \%$ amino acid homology in the extracellular portion with other B7 family members. The mouse and human amino acid sequences of B7-H4 share approximately $87 \%$ amino acid identity [2]. B7-H4 mRNA is widely expressed in human peripheral tissues, including lung, testis, pancreas, prostate, placenta, uterus, skin, muscle, intestine, stomach, kidney, liver, heart, brain, and ovary [1-3]. However, its protein expression on tissues seems to be limited [2, 4]. Initially, B7-H4 expression was observed in cancer cells of colon, prostate, lung, and fibrosarcoma $[3,5]$, and human ovarian and lung cancer tissues [4]. Subsequent studies from different laboratories have demonstrated that the expression of B7-H4 mRNA and protein was detected in all of the 23 melanoma cell lines [6], 5 gastric cancer cell lines [7], and 6 non-small-cell lung cancer cell lines [8]. To date, B7-H4 expression has been found in many different types of human cancer tissues, and soluble B7-H4 has also been detected in blood samples from cancer patients. The expression pattern of B7-H4 in human cancer tissues and its clinical significances will be discussed in Section 4.

B7-H4 is not expressed in naïve T and B cells, but after stimulation by interleukin-6 (IL-6) and IL-10, B7-H4 is inducibly expressed in APCs, including dendritic cells (DCs), monocytes and macrophages [1-3]. On the other hand, the DC-differentiation cytokines, granulocyte/macrophage colony-stimulating factor (GM-CSF) and IL-4, decrease the expression of $\mathrm{B} 7-\mathrm{H} 4$ in these cells [9-11]. However, interferons (INFs) appear to have minimal effects on the induction of B7-H4 expression [9-11].

In human ovarian cancer, tumor-associated regulatory $\mathrm{T}$ (Treg) cells trigger macrophages to produce IL-6 and IL-10, and these cytokines in turn stimulate APCs to express B7-H4 in an autocrine and/or paracrine manner [9]. High levels of IL- 6 and IL-10, but not GM-CSF and IL-4, are detected in the ovarian tumor microenvironment. Therefore, this dysfunctional cytokine network in the tumor microenvironment may enable APCs to express B7-H4. Interestingly, IL-4, IL-6, IL-10 and GM-CSF have no regulatory effects on the expression of $\mathrm{B} 7-\mathrm{H} 4$ on tumor cells, indicating that the expression of B7-H4 in tumor cells may be functionally distinct and differently regulated compared with APCs $[9,12]$.

To date, the receptor of $\mathrm{B} 7-\mathrm{H} 4$ has not yet been identified. B and T lymphocyte attenuator (BTLA) was initially proposed to be the receptor for $\mathrm{B} 7-\mathrm{H} 4$ [5], but further studies have not supported this proposal, as BTLA has not shown to directly bind to B7-H4 but may influence the appearance of an unknown receptor for B7-H4 on the Th1 cell surface [13$15]$.

\section{Negative Effects of B7-H4 on Antitumor Immunity}

3.1. Adaptive Immunity. B7-H4 inhibits the activation, proliferation, clonal expansion of $\mathrm{CD} 4^{+}$and $\mathrm{CD} 8^{+} \mathrm{T}$-cells, thus suppressing the production of cytokines (IL-2, IFN- $\gamma$ ), and generation of alloreactive cytotoxic $\mathrm{T}$ lymphocytes (CTLs) by arresting the cell cycle in an in vitro T-cell activation assay $[1,2,5]$. B7-H4 expressed on the surface of surrogate APCs also inhibits the proliferation of $\mathrm{T}$ cells $[2,5]$. In vivo blockade of endogenous $\mathrm{B} 7-\mathrm{H} 4$ by a specific $\mathrm{mAb}$ promoted T-cell response, indicating that $\mathrm{B} 7-\mathrm{H} 4$ plays an inhibitory role in T-cell activation [2]. The inhibitory effects of $\mathrm{B} 7-\mathrm{H} 4$ on T-cell activation and proliferation are also supported by the finding that acute lymphopenia-induced homeostatic proliferation of $\mathrm{T}$ cells promotes antitumor immunity. However, these cells display a severe deficit in the expression of $\mathrm{B} 7-\mathrm{H} 4$ as they show lower suppression by a specific Ab against B7-H4 and fail to produce IL-10 [16]. B7$\mathrm{H} 4$-deficient Balb/c mice mounted mildly augmented Th1 responses and displayed slightly lower parasite burdens upon Leishmania major infection compared to the wild-type mice, indicating that $\mathrm{B} 7-\mathrm{H} 4$ could inhibit Th1 response against infection [17]. However, the lack of B7-H4 did not affect hypersensitive inflammatory responses in the airway or skin that are induced by either Th1 or Th2 cells. Likewise, B7H4-deficient mice developed normal CTL reaction against viral infection [17]. These results suggest that B7-H4 may be one of multiple negative cosignaling molecules that collectively provide a fine-tuning mechanism for T-cellmediated immune responses [18].

There is no direct evidence of a barrier function for B7$\mathrm{H} 4$, although it is variably glycosylated in tumor-specific patterns, suggesting that glycosylation may be a potential mechanism for modulating interaction of CTLs with tumor cells [19]. A physical blockade would complement the ability of B7-H4, when ligated to its unknown receptor on T cells, to inhibit cytokine secretion, and proliferation of $\mathrm{T}$ cells predominantly through cell cycle arrest [2].

The effects of $\mathrm{B} 7-\mathrm{H} 4$ on $\mathrm{B}$ cells have not been investigated. However, enhanced B7-H4 expression on B cells infected with Epstein-Barr virus (EBV) increased the levels of intracellular reactive oxygen species (ROS), induced the expression of Fas ligand, and subsequently led to Fasmediated and caspase-dependent apoptosis in association with increased release of cytochrome c, apoptosis-inducing factor (AIF), and EndoG from the mitochondria [20]. In a subsequent study by the same group, engagement of B7-H4 significantly reduced cell growth of EBV-positive lymphoma cells, resulting in cell cycle arrest at G0-G1 phase via downregulation of CDK4/6, CDK2, cyclin E/D expression, phosphor-AKT, and phosphor-cyclin $\mathrm{E}$ and upregulation of p21 expression [21]. These results suggest that B7-H4 may be a potential target for EBV-positive lymphoma therapy. Although not investigated, these studies may also imply that B7-H4 could inhibit proliferation and activation, and induce apoptosis of $\mathrm{B}$ cells, thus impairing the production of immunoglobulins and contributing to the suppression of adaptive immunity.

In addition, it has been demonstrated that tumoral B7- $\mathrm{H} 4^{+}$macrophages and $\mathrm{CD} 4^{+} \mathrm{CD} 25^{+} \mathrm{FoxP}^{+}$Treg cells suppressed tumor-associated antigen-specific T-cell immunity [10]. The tumor-associated macrophages spontaneously produce chemokine CCL22 to mediate Treg cell trafficking into tumors, and Treg cells induce the expression of B7-H4 on APCs and macrophages [10]. It has been shown that Treg cells induced macrophages to spontaneously produce IL-10 
and IL-6, which in turn stimulated B7-H4 expression on macrophages in an autocrine manner through IL-10 and IL6 [9]. The two studies suggest that Treg cells may convey their suppressive activity to APCs through B7-H4 induction [9].

3.2. Innate Immunity. To date, there has been only one published study [22], which has investigated the role of B7-H4 in innate immunity. It has been shown that the inhibitory effect of B7-H4 on innate immunity was mediated through controlling the growth of neutrophils [22]. B7-H4 knockout mice were more resistant to infection by Listeria monocytogenes than their littermates, suggesting that B7H4 plays an inhibitory role on innate immunity. Further studies have shown that more neutrophils were observed in peripheral organs of $\mathrm{B} 7-\mathrm{H} 4$ knockout mice than their littermates but their bactericidal functions remained unchanged. In vitro, B7-H4 inhibited the growth of bone marrowderived neutrophil progenitors, suggesting an inhibitory function of B7-H4 in neutrophil expansion. As augmented innate resistance is completely dependent on neutrophils, even in the absence of adaptive immunity, the results indicate that $\mathrm{B} 7-\mathrm{H} 4$ serves as a negative regulator of the neutrophil response to infection, and provides a new target for manipulation of innate immunity.

3.3. Cancer Immunity. B7-H4 has been found to be expressed at the mRNA and protein levels in many types of human cancers and negatively correlate with poor prognosis (Refer to Section 4). Expression of B7-H4 in human tumors is most likely due to aberrant regulation of posttranscription in tumors, since its cell surface protein expression is rare in normal human tissues, though abundant B7-H4 mRNA is detected [18]. B7-H4 was preferentially expressed in nondividing tumor cells from human gliomas and medulloblastomas, and in a subset of brain tumor stem-like $\mathrm{CD}_{133^{+}}$cells $[23]$. The $\mathrm{CD} 133^{+}$cell-initiated glioblastomas showed a higher proliferation index than $\mathrm{CD} 133^{-}$cellinduced glioblastomas in immune-deficient mice [23].

Increased B7-H4 expression in tumor cells correlated with decreased cell apoptosis and enhanced outgrowth of tumors in several models, including the severe combined immunodeficiency (SCID)/Beige xenograft outgrowth model [22]. B7-H4 has also been shown to be extensively and variably N-glycosylated, which may serve as a "barrier" mechanism to evade immunosurveillance [22]. As suggested by $\mathrm{Yi}$ and $\mathrm{Chen}$, the role of $\mathrm{B} 7-\mathrm{H} 4$ in tumor progression may be to transform precancerous cells and then protect them from immunosurveillance [18]. In addition, one study has shown that overexpression of $\mathrm{B} 7-\mathrm{H} 4$ promoted tumorigenesis of ovarian cancer in immunodeficient mice by increased proliferation rate, cell adhesion, migration, and invasion [24], implying that B7-H4 might have a direct effect on tumorigenesis independent of immunity. In another study, overexpression of B7-H4 on normal cells resulted in malignant cellular transformation of epithelial cells, perhaps by protecting the pretransformed cells from apoptosis, as siRNA knockdown of B7-H4 on tumor cell lines in vitro led to increased apoptosis [19]. However, the direct effect of
B7-H4 on tumorigenesis has been only demonstrated in the above two studies, thus the exact mechanisms need further investigation.

In the tumor microenvironment, in addition to tumor cells, tumor-infiltrating macrophages $[9,10]$ and endothelial cells of small blood vessels [12] have also been found to constitutively express B7-H4. B7-H4 was highly expressed in tumor-associated macrophages in the ascites of ovarian cancer patients and contributed to tumor progression [10]. B7-H4 blockade by antisense oligonucleotides restored the function of macrophages to stimulate $\mathrm{T}$ cells and led to tumor regression in vivo $[10,11]$.

\section{B7-H4 Expression in Human Cancers and Its Significance}

In the present paper, the clinical data in support of the possible function of B7-H4 in antitumor immunity come from 26 retrospective analyses on 13 types of human cancers including the most common ones, that is, cancers of ovary, esophagus, kidney, stomach, liver, lung, colon, pancreas, breast and prostate, and melanoma. All relevant studies on the expression of B7-H4 on human cancer tissues or levels of soluble B7-H4 in human blood samples and the clinical significance are summarized in Table $1[4-9,12,19,23,25-$ 41]. A negative correlation between $\mathrm{B} 7-\mathrm{H} 4$ expression and Tcell infiltration has been reported [25, 32, 34]. However, such correlation was not observed in a study on melanoma [6].

The expression of B7-H4 has been most widely studied in ovarian cancer. To date, ten studies have investigated the expression of $\mathrm{B} 7-\mathrm{H} 4$ in ovarian cancer tissues and/or the level of soluble B7-H4 in blood samples from the ovarian cancer patients $[4,9,19,23,28-30,35-38]$. The positive B7-H4 expression rates in ovarian cancer tissues range from 9 to $100 \%$ as shown by immunohistochemistry. Most of the studies have revealed the correlation between expression levels of B7-H4 and survival, pathological types, or tumor TNM staging. The levels of soluble B7-H4 in blood correlate with tumor stage, poor prognosis, and pathological types, indicating that B7-H4 may be a potential diagnostic marker and a prognostic predictor for ovarian cancer. However, one study did not show the similar correlation between soluble B7-H4 levels in blood and other diagnostic markers for ovarian cancer patients [27].

Breast cancer is the second most studied cancer for B7H4 expression. To date, six studies have investigated the expression of B7-H4 in human breast cancer tissues [19, 30, $32,33,37]$, but two of which lack detailed data. In one study, 193 primary breast tumors and 246 metastatic breast tumors were examined by immunohistochemistry and the B7-H4 positive expression rates were as high as $95.4 \%$ in primary tumors and $97.6 \%$ in metastatic tumors, and the increased expression of $\mathrm{B} 7-\mathrm{H} 4$ correlated with negative progesterone receptor and HER-2/neu status [33]. Similarly, the other two studies have demonstrated a positive expression rate of B7-H4 mRNA and protein at 100\%, determined by reverse transcription polymerase chain reaction (RT-PCR) [41] and immunohistochemistry [19], respectively. 


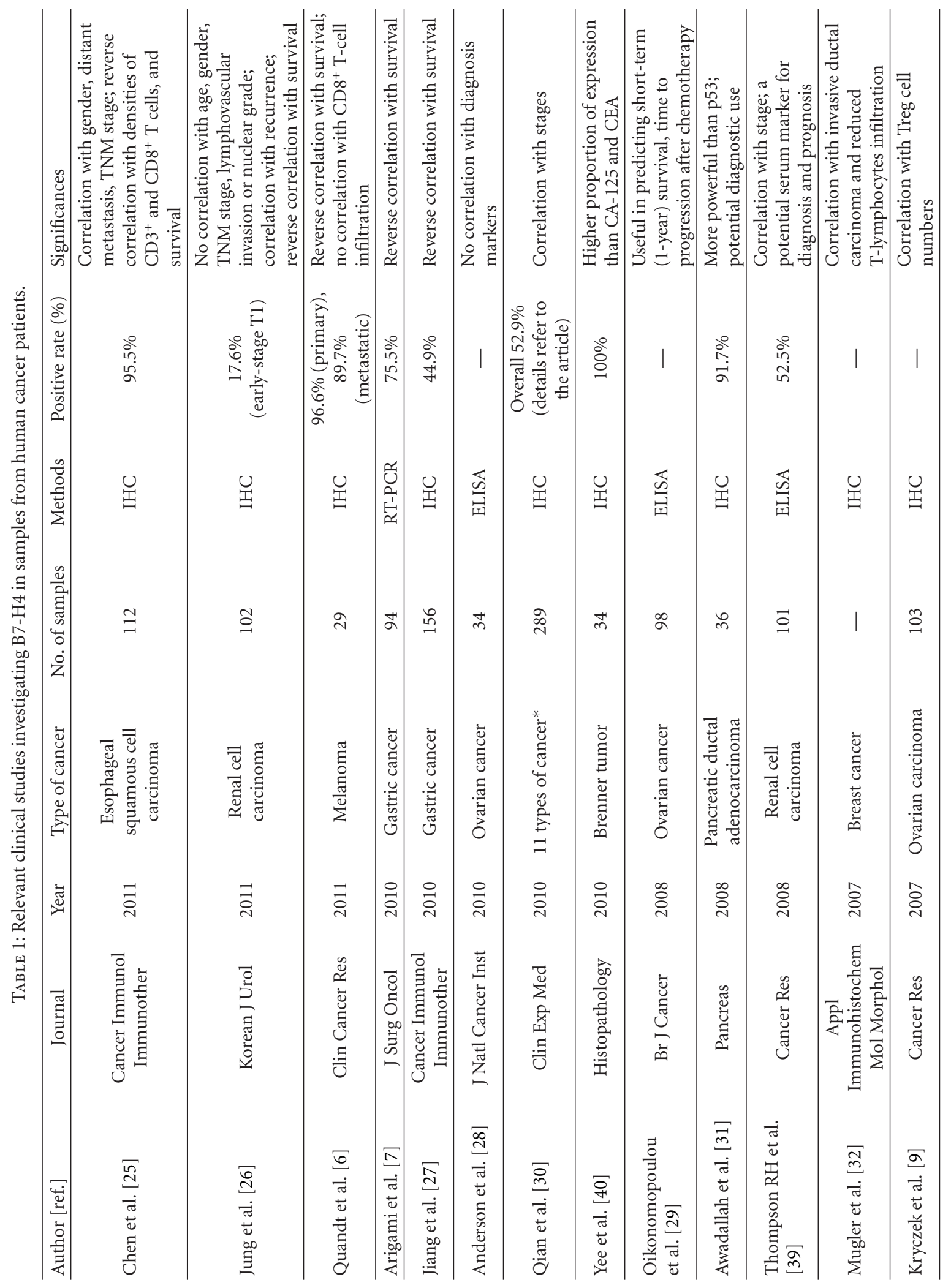




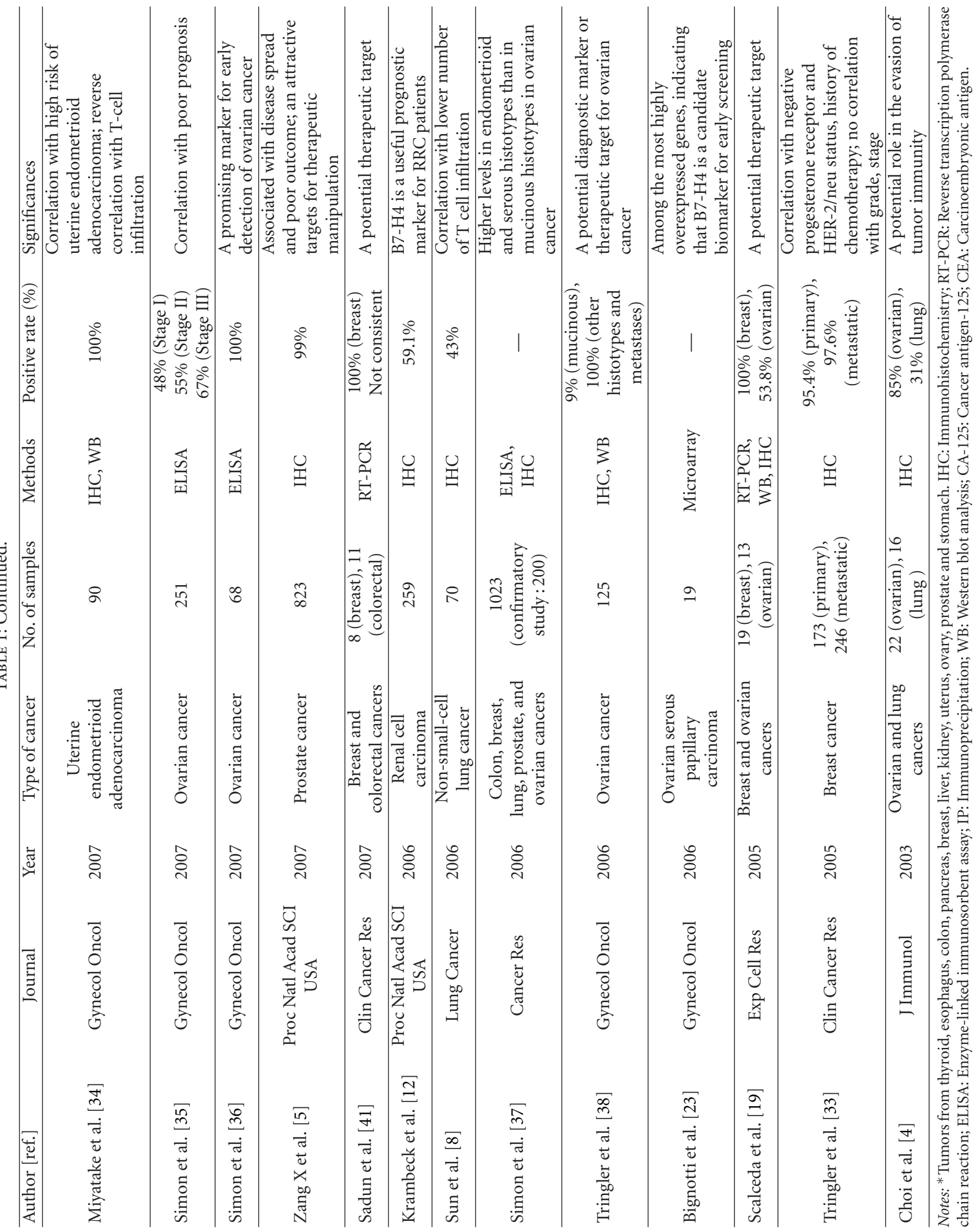


In two studies on lung cancer, $31 \%$ [4] and $43 \%$ [8] of lung cancer tissues were found to express B7-H4 detected by immunohistochemistry, respectively. In a study with 259 cases of renal cell carcinoma (RCC), $59.1 \%$ of the cancer tissues had B7-H4 protein expression [12]. However, the B7$\mathrm{H} 4$ positive expression rate was found to be only $17.6 \%$ in 102 cases of early-stage RCC (T1), and B7-H4 expression did not correlate with age, gender, TNM stage, lymphovascular invasion, or nuclear grade, but correlated with cancer recurrence and negatively correlated with survival [26]. 75.5\% (71/94) of gastric cancer tissues were found to express $\mathrm{B} 7-\mathrm{H} 4$ mRNA [7], but the B7-H4 protein positive expression rate detected by immunohistochemistry dropped to $44.9 \%$ in another study with 156 cases of gastric cancer [27]. In one study with 24 cases of gastric cancer, the positive rate was as low as only $12.5 \%$. Although not widely investigated, over $90 \%$ of the tissues from melanoma [6], pancreatic ductal adenocarcinoma [31], uterine cancer [30, 34], esophageal squamous cell carcinoma [25], and prostate cancer [5] expressed B7-H4, shown by immunohistochemistry. Although one study has reported that $63.6 \%(14 / 22)$ of colon cancer tissues expressed B7-H4 [30], the expression of B7-H4 was found to be less consistent $[37,41]$. B7-H4 was found to be expressed in 100\% (34/34) of Brenner tumors [40]. Although Brenner tumors are of benign feature, this report is exceptionally included in Table 1.

Soluble B7-H4 was detected in blood samples from patients of ovarian cancer, RCC, colon cancer, breast cancer, lung cancer, and prostate cancer [8, 28, 29, 35-37, 39]. These studies indicate that serum B7-H4 may be a useful marker for diagnosis and prognosis, but the mechanism of production and the function of soluble B7-H4 remains unknown.

\section{Potential of B7-H4 in Clinical Application}

Because of the higher expression of $\mathrm{B} 7-\mathrm{H} 4$ in cancer tissues compared with corresponding normal tissues and its close correlation with stage, pathological types and biological behavior of tumors, and survival of cancer patients, we should pay attention to the potential diagnostic and prognostic capacities of B7-H4 for identifying cancer, determining pathologic variables, and predicting response to treatment and survival. We believe that B7-H4 could become potent tools to add to the oncologist's toolbox for early diagnosing cancer, monitoring the efficacy of treatments and predicting the prognosis. This may be possible when the expression patterns of B7-H4 have been investigated on a larger number of samples from different types of cancers and from multiple centers.

Given that B7-H4 is highly expressed in almost all the examined cell lines from cancers of colon, prostate, lung, and stomach, and fibrosarcoma and melanomas [38 ] and in various human cancer tissues (Table 1), it could be hypothesized that the expression of $\mathrm{B} 7-\mathrm{H} 4$ represents a mechanism of downregulating antitumor immunity, particularly T-cell response, at the level of the effector cells [5]. This paradigm calls for the development of new strategies for tumor immunotherapy by targeting B7-H4 [42].
B7-H4 inhibits T-cell function $[1-3,5]$, indicating that B7H4-positive tumor cells have an advantage over the B7H4-negative tumor cells by downregulating T-cell-mediated antitumor immunity. Consequently, the blockade of tumorassociated B7-H4 could offer a new therapeutic opportunity for enhancing antitumor immunity. Efficient neutralizing antibodies specific for human B7H4 are not yet available. Small interfering RNA (siRNA) [19] and antisense oligonucleotides specific for $\mathrm{B} 7-\mathrm{H} 4[10,11]$ have been used to block B7-H4 expression. Blocking the expression of B7-H4 in tumor-associated macrophages disabled their suppressive capacity, enabled tumor-associated antigen- (TAA-) specific effector $\mathrm{T}$ cells function, and suppressed tumor growth in human ovarian cancer xenografts $[10,11]$. In addition, the expression of B7-H4 on endothelial cells of tumor vasculature has also been observed in RCC tissues [12]. Although the mechanism accounting for what signals trigger B7-H4 expression in tumor vessels remains unknown, one most likely source could be the tumor microenvironments. Tumor blood vessels are distinct from normal resting blood vessels, and can be selectively destroyed without significantly affecting normal vessels. Therefore, blockade and/or destruction of tumor vasculature-associated B7-H4 might provide a dual beneficial therapy, that is, enhancement of $\mathrm{T}$ cell-mediated antitumor immunity and destruction of tumor vessels.

\section{Conclusions and Future Prospects}

Recent data indicate that B7-H4 functions in peripheral tissues to negatively regulate immune responses in target organs. While its broad distribution is observed at mRNA level, limited expression at the protein level suggests that tight control of B7-H4 is imposed at posttranscriptional level. Receptor identification remains the manifest topic and is critical for understanding the role of B7-H4, as it is certainly essential to understand the complex role, but continues to be difficult due primarily to low receptor/ligand affinities. Therefore, more studies are required to seek and identify the receptor for B7-H4. Increased B7-H4 expression in tumor tissues and high levels in blood samples of cancer patients represent a realistic opportunity to design novel immunotherapeutic approaches by regulating the immune response through manipulating the expression of $\mathrm{B} 7-\mathrm{H} 4$ and/or its receptor. B7-H4 can also serve as a useful biomarker for cancer diagnosis and prognosis prediction, when its expression patterns have been further investigated.

\section{Acknowledgment}

This study was supported by Grants from the National Natural Scientific Foundation of China (30872987 and 30973474).

\section{References}

[1] D. V. R. Prasad, S. Richards, X. M. Mai, and C. Dong, "B7S1, a novel B7 family member that negatively regulates $\mathrm{T}$ cell activation," Immunity, vol. 18, no. 6, pp. 863-873, 2003. 
[2] G. L. Sica, I. H. Choi, G. Zhu et al., "B7-H4, a molecule of the $\mathrm{B} 7$ family, negatively regulates $\mathrm{T}$ cell immunity," Immunity, vol. 18, no. 6, pp. 849-861, 2003.

[3] X. Zang, P. Loke, J. Kim, K. Murphy, R. Waitz, and J. P. Allison, "B7x: a widely expressed B7 family member that inhibits T cell activation," Proceedings of the National Academy of Sciences of the United States of America, vol. 100, no. 18, pp. 10388-10392, 2003.

[4] I. H. Choi, G. Zhu, G. L. Sica et al., "Genomic organization and expression analysis of $\mathrm{B} 7-\mathrm{H} 4$, an immune inhibitory molecule of the B7 family," Journal of Immunology, vol. 171, no. 9, pp. 4650-4654, 2003.

[5] X. Zang, R. H. Thompson, H. A. Al-Ahmadie et al., "B7-H3 and $\mathrm{B} 7 \mathrm{x}$ are highly expressed in human prostate cancer and associated with disease spread and poor outcome," Proceedings of the National Academy of Sciences of the United States of America, vol. 104, no. 49, pp. 19458-19463, 2007.

[6] D. Quandt, E. Fiedler, D. Boettcher, W. Ch. Marsch, and B. Seliger, "B7-H4 expression in human melanoma: its association with patients' survival and antitumor immune response," Clinical Cancer Research, vol. 17, no. 10, pp. 3100$3111,2011$.

[7] T. Arigami, Y. Uenosono, M. Hirata et al., "Expression of B7$\mathrm{H} 4$ in blood of patients with gastric cancer predicts tumor progression and prognosis," Journal of Surgical Oncology, vol. 102, no. 7, pp. 748-752, 2010.

[8] Y. Sun, Y. Wang, J. Zhao et al., "B7-H3 and B7-H4 expression in non-small-cell lung cancer," Lung Cancer, vol. 53, no. 2, pp. 143-151, 2006.

[9] I. Kryczek, S. Wei, G. Zhu et al., "Relationship between B7$\mathrm{H} 4$, regulatory $\mathrm{T}$ cells, and patient outcome in human ovarian carcinoma," Cancer Research, vol. 67, no. 18, pp. 8900-8905, 2007.

[10] I. Kryczek, L. Zou, P. Rodriguez et al., "B7-H4 expression identifies a novel suppressive macrophage population in human ovarian carcinoma," Journal of Experimental Medicine, vol. 203, no. 4, pp. 871-881, 2006.

[11] I. Kryczek, S. Wei, L. Zou et al., "Cutting edge: induction of B7-H4 on APCs through IL-10: novel suppressive mode for regulatory T cells," Journal of Immunology, vol. 177, no. 1, pp. 40-44, 2006.

[12] A. E. Krambeck, R. H. Thompson, H. Dong et al., "B7-H4 expression in renal cell carcinoma and tumor vasculature: associations with cancer progression and survival," Proceedings of the National Academy of Sciences of the United States of America, vol. 103, no. 27, pp. 10391-10396, 2006.

[13] D. M. Compaan, L. C. Gonzalez, I. Tom, K. M. Loyet, D. Eaton, and S. G. Hymowitz, "Attenuating lymphocyte activity: the crystal structure of the BTLA-HVEM complex," Journal of Biological Chemistry, vol. 280, no. 47, pp. 39553-39561, 2005.

[14] L. C. Gonzalez, K. M. Loyet, J. Calemine-Fenaux et al., "A coreceptor interaction between the CD28 and TNF receptor family members B and T lymphocyte attenuator and herpesvirus entry mediator," Proceedings of the National Academy of Sciences of the United States of America, vol. 102, no. 4, pp. 1116-1121, 2005.

[15] J. R. Sedy, M. Gavrieli, K. G. Potter et al., "B and T lymphocyte attenuator regulates $\mathrm{T}$ cell activation through interaction with herpesvirus entry mediator," Nature Immunology, vol. 6, no. 1, pp. 90-98, 2005.

[16] A. Shvets, R. Chakrabarti, R. Gonzalez-Quintial, R. Baccala, A. N. Theofilopoulos, and G. J. Prudhomme, "Impaired negative regulation of homeostatically proliferating T cells," Blood, vol. 113, no. 3, pp. 622-625, 2009.
[17] W. K. Suh, S. Wang, G. S. Duncan et al., "Generation and characterization of B7-H4/B7S1/B7x-deficient mice," Molecular and Cellular Biology, vol. 26, no. 17, pp. 6403-6411, 2006.

[18] K. H. Yi and L. Chen, "Fine tuning the immune response through B7-H3 and B7-H4," Immunological Reviews, vol. 229, no. 1, pp. 145-151, 2009.

[19] S. Salceda, T. Tang, M. Kmet et al., "The immunomodulatory protein $\mathrm{B} 7-\mathrm{H} 4$ is overexpressed in breast and ovarian cancers and promotes epithelial cell transformation," Experimental Cell Research, vol. 306, no. 1, pp. 128-141, 2005.

[20] H. Song, G. Park, Y. S. Kim et al., "B7-H4 reverse signaling induces the apoptosis of EBV-transformed B cells through Fas ligand up-regulation," Cancer Letters, vol. 266, no. 2, pp. 227237, 2008.

[21] G. B. Park, H. Song, Y. S. Kim et al., "Cell cycle arrest induced by engagement of B7-H4 on Epstein-Barr virus-positive B-cell lymphoma cell lines," Immunology, vol. 128, no. 3, pp. 360368, 2009.

[22] G. Zhu, M. M. Augustine, T. Azuma et al., "B7-H4-deficient mice display augmented neutrophil-mediated innate immunity," Blood, vol. 113, no. 8, pp. 1759-1767, 2009.

[23] E. Bignotti, R. A. Tassi, S. Calza et al., "Differential gene expression profiles between tumor biopsies and short-term primary cultures of ovarian serous carcinomas: identification of novel molecular biomarkers for early diagnosis and therapy," Gynecologic Oncology, vol. 103, no. 2, pp. 405-416, 2006.

[24] L. Cheng, J. Jiang, R. Gao et al., "B7-H4 expression promotes tumorigenesis in ovarian cancer," International Journal of Gynecological Cancer, vol. 19, no. 9, pp. 1481-1486, 2009.

[25] L. -J. Chen, J. Sun, H. Y. Wu et al., "B7-H4 expression associates with cancer progression and predicts patient's survival in human esophageal squamous cell carcinoma," Cancer Immunology, Immunotherapy, vol. 60, no. 7, pp. 10471055, 2011.

[26] S. G. Jung, K. U. Choi, S. D. Lee, Z. Z. Lee, and M. K. Chung, "The relationship between B7-H4 expression and clinic pathological characteristics in clinical stage T1 conventional renal cell carcinoma," Korean Journal of Urology, vol. 52, no. 2, pp. 90-95, 2011.

[27] J. Jiang, Y. Zhu, C. Wu et al., "Tumor expression of B7$\mathrm{H} 4$ predicts poor survival of patients suffering from gastric cancer," Cancer Immunology, Immunotherapy, vol. 59, no. 11, pp. 1707-1714, 2010.

[28] G. L. Anderson, M. McIntosh, L. Wu et al., "Assessing lead time of selected ovarian cancer biomarkers: a nested case-control study," Journal of the National Cancer Institute, vol. 102, no. 1, pp. 26-38, 2010.

[29] K. Oikonomopoulou, L. Li, Y. Zheng et al., "Prediction of ovarian cancer prognosis and response to chemotherapy by a serum-based multiparametric biomarker panel," British Journal of Cancer, vol. 99, no. 7, pp. 1103-1113, 2008.

[30] Y. Qian, L. Shen, L. Cheng, Z. Wu, and H. Yao, "B7$\mathrm{H} 4$ expression in various tumors determined using a novel developed monoclonal antibody," Clinical and Experimental Medicine, vol. 11, no. 3, pp. 163-170, 2010.

[31] N. S. Awadallah, K. R. Shroyer, D. A. Langer et al., "Detection of B7-H4 and p53 in pancreatic cancer: potential role as a cytological diagnostic adjunct," Pancreas, vol. 36, no. 2, pp. 200-206, 2008.

[32] K. C. Mugler, M. Singh, B. Tringler et al., "B7-H4 expression in a range of breast pathology: correlation with tumor $\mathrm{T}$ cell infiltration," Applied Immunohistochemistry and Molecular Morphology, vol. 15, no. 4, pp. 363-370, 2007. 
[33] B. Tringler, S. Zhuo, G. Pilkington et al., "B7-H4 is highly expressed in ductal and lobular breast cancer," Clinical Cancer Research, vol. 11, no. 5, pp. 1842-1848, 2005.

[34] T. Miyatake, B. Tringler, W. Liu et al., "B7-H4 (DD-O110) is overexpressed in high risk uterine endometrioid adenocarcinomas and inversely correlated with tumor T-cell infiltration," Gynecologic Oncology, vol. 106, no. 1, pp. 119-127, 2007.

[35] I. Simon, D. Katsaros, I. Rigault de la Longrais et al., "B7$\mathrm{H} 4$ is over-expressed in early-stage ovarian cancer and is independent of CA125 expression," Gynecologic Oncology, vol. 106, no. 2, pp. 334-341, 2007.

[36] I. Simon, Y. Liu, K. L. Krall et al., "Evaluation of the novel serum markers B7-H4, Spondin 2, and DcR3 for diagnosis and early detection of ovarian cancer," Gynecologic Oncology, vol. 106, no. 1, pp. 112-118, 2007.

[37] I. Simon, S. Zhuo, L. Corral et al., "B7-H4 Is a novel membrane-bound protein and a candidate serum and tissue biomarker for ovarian cancer," Cancer Research, vol. 66, no. 3, pp. 1570-1575, 2006.

[38] B. Tringler, W. Liu, L. Corral et al., "B7-H4 overexpression in ovarian tumors," Gynecologic Oncology, vol. 100, no. 1, pp. 4452, 2006.

[39] R. H. Thompson, X. Zang, C. M. Lohse et al., "Serumsoluble B7x is elevated in renal cell carcinoma patients and is associated with advanced stage," Cancer Research, vol. 68, no. 15, pp. 6054-6058, 2008.

[40] E. U. Yee, R. J. Zaino, K. C. Torkko, and K. R. Shroyer, "B7-H4 expression in brenner tumours, a descriptive and comparative study," Histopathology, vol. 56, no. 5, pp. 652-654, 2010.

[41] R. E. Sadun, S. M. Sachsman, X. Chen et al., "Immune signatures of murine and human cancers reveal unique mechanisms of tumor escape and new targets for cancer immunotherapy," Clinical Cancer Research, vol. 13, no. 13, pp. 4016-4025, 2007.

[42] E. Pure, J. P. Allison, and R. D. Schreiber, "Breaking down the barriers to cancer immunotherapy," Nature Immunology, vol. 6, no. 12, pp. 1207-1210, 2005. 


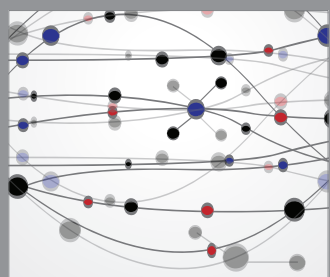

The Scientific World Journal
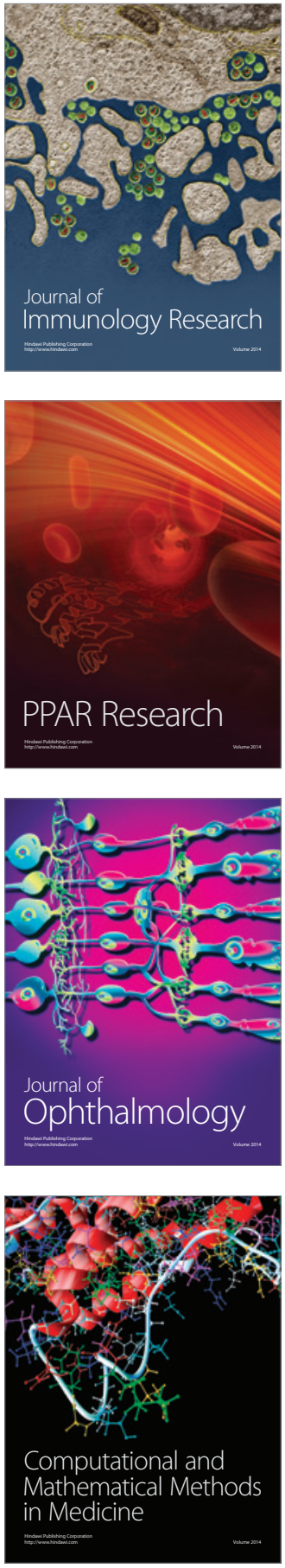

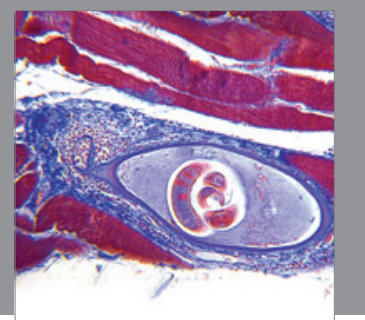

Gastroenterology

Research and Practice
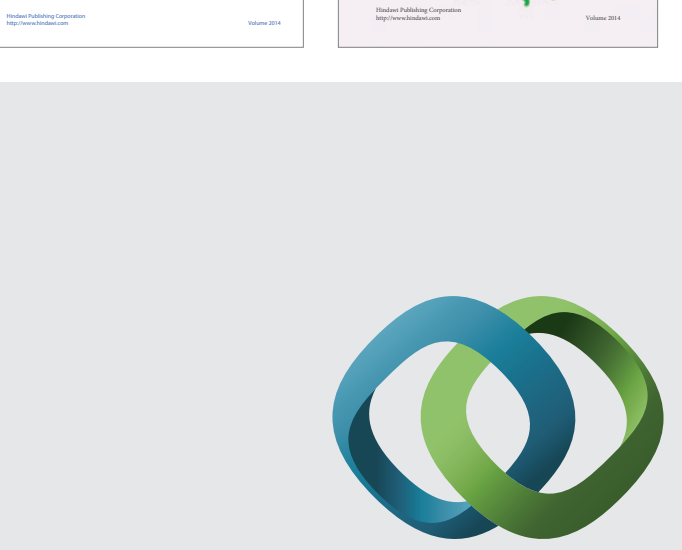

\section{Hindawi}

Submit your manuscripts at

http://www.hindawi.com
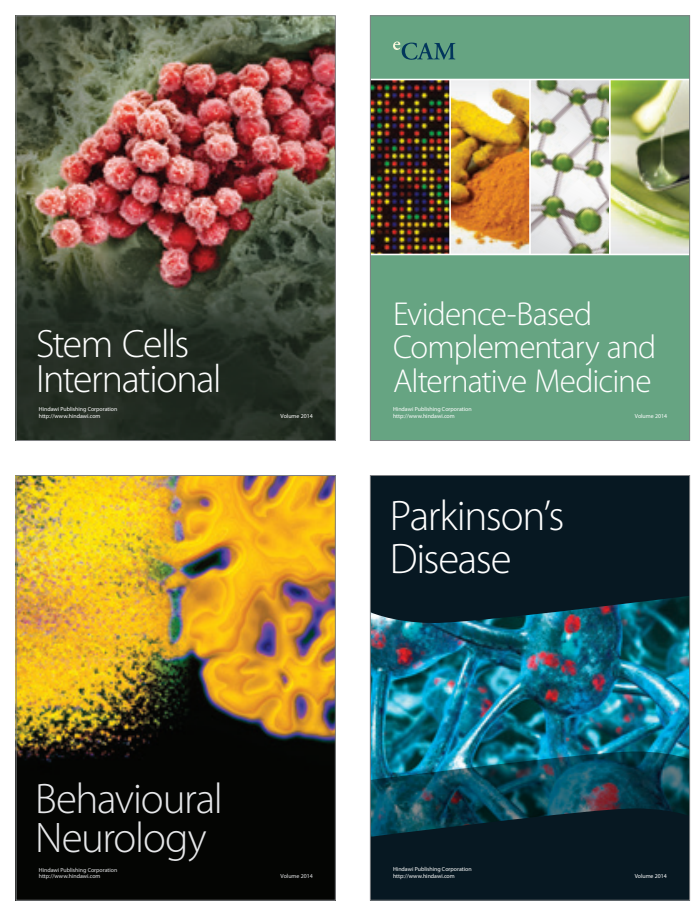

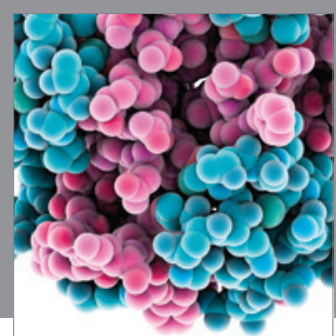

Journal of
Diabetes Research

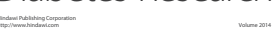

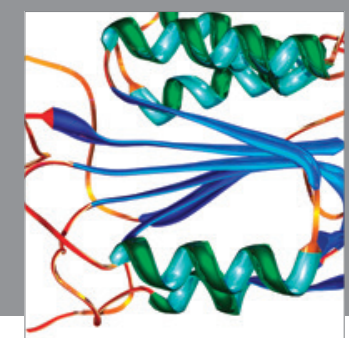

Disease Markers
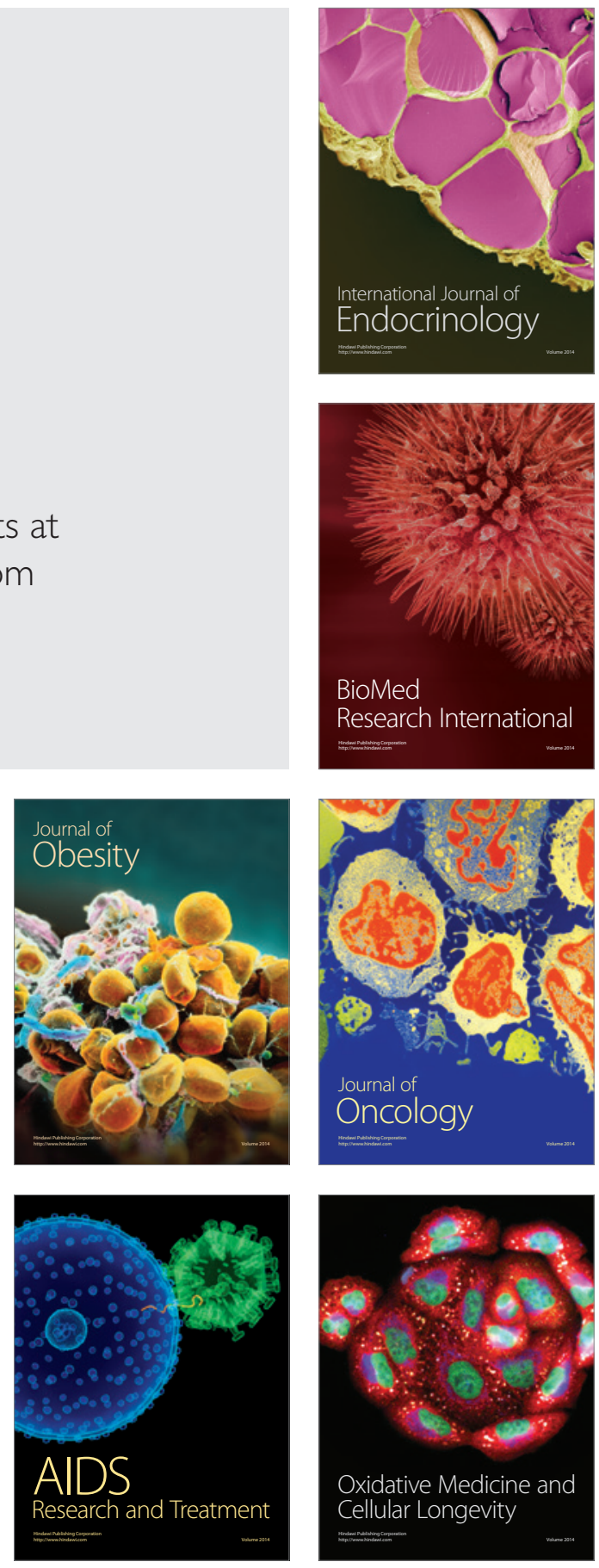